In September 2019, NIA and NIEHS launched the Telomere Research Network (TRN) (trn.tulane.edu) to establish best practices for the measurement of telomere length in population-based studies. As a first step, this working document was drafted to reflect the current recommendations of the TRN. These recommendations are offered as initial guidelines for researchers, reviewers, and scientific research officers, and are considered minimal reporting guidelines for PCR-based measurement of telomere length. Over the next five years, the TRN expects to better define these parameters and integrate comments from our colleagues and experts around the world. To make comments and/or request clarification please contact Stacy Drury, M.D., PhD, Director of the TRN at telomerenetwork@gmail.com.

\title{
Minimum Reporting Recommendations for PCR-based Telomere Length Measurement
}

\section{Sample type, storage, extraction and integrity:}

Sample type ${ }^{1}$

Sample storage conditions, including temperature, duration, and buffer ${ }^{2,3}$

DNA extraction method ${ }^{4}$

DNA storage conditions, including freeze-thaw cycles $^{5,6,7}$

Method of documenting DNA quality and integrity ${ }^{8}$

Percentage of samples specifically tested for DNA quality and integrity

For studies with repeated measures design, report the above for all time points

\section{qPCR assay:}

State whether qPCR, MMqPCR, aTL (absolute TL/PCR based) or other PCR based method PCR machine type ${ }^{9}$

Source (manufacturer/home-made) of master mix and reagents, and final reaction volume ${ }^{10}$

Telomere primer sequences and concentration ${ }^{11}$

Single copy gene name, primer sequences, and concentration ${ }^{11}$

Full PCR program description including temperature, times, and cycle numbers ${ }^{11}$

PCR efficiency of single copy gene and telomere primers

Source and concentration of control samples and standard curve ${ }^{11}$

For aTL PCR measurement only: sequence and concentration of oligo standards

\section{Data analysis:}

Mean and standard deviation or median and range of telomere lengths

Number of sample replicates

Level of independence of the replicates (plate vs day vs extraction)

Analytic method, considering replicate measurements, to determine final telomere length ${ }^{12}$

Method of accounting for variation between sample replicates

Method for accounting for well position effects within plates ${ }^{12}$

Method of accounting for between plate effects ${ }^{12}$

$\%$ of samples repeated and \% samples failing final QC and excluded from further analyses

Acceptable range of PCR efficiency for the single copy gene and telomere primers

ICCs of sample/study groups to address variability (not CV) ) $^{13,14}$

$\mathrm{T} / \mathrm{S}$ ratio transformed to a $\mathrm{z}$ score prior before comparison across methods/studies ${ }^{15}$

For studies with family samples or repeated measure design: analytic method to account for this ${ }^{16,17}$

Note: Currently, we do NOT recommend transformation of T/S measurement to base pairs for qPCR/MMPqPCR assays. 
References:

1. Dlouha D, Maluskova J, Kralova Lesna I, Lanska V, Hubacek JA. Comparison of the relative telomere length measured in leukocytes and eleven different human tissues. Physiol Res. 2014;63 Suppl 3:S343-S350.

2. Zanet DL, Saberi S, Oliveira L, Sattha B, Gadawski I, Côté HC. Blood and dried blood spot telomere length measurement by qPCR: assay considerations. PLoS One. 2013;8(2):e57787. doi:10.1371/journal.pone.0057787

3. Kong PL, Looi LM, Lau TP, Cheah PL. Assessment of Telomere Length in Archived Formalin-Fixed, Paraffinized Human Tissue Is Confounded by Chronological Age and Storage Duration [published correction appears in PLoS One. 2016 Dec 9;11(12 ):e0168238]. PLoS One. 2016;11(9):e0161720. Published 2016 Sep 6. doi:10.1371/journal.pone.0161720

4. Cunningham JM, Johnson RA, Litzelman K, et al. Telomere length varies by DNA extraction method: implications for epidemiologic research. Cancer Epidemiol Biomarkers Prev. 2013;22(11):2047-2054. doi:10.1158/1055-9965.EPI-13-0409

5. Dagnall CL, Hicks B, Teshome K, Hutchinson AA, Gadalla SM, et al. (2017) Effect of pre-analytic variables on the reproducibility of qPCR relative telomere length measurement. PLOS ONE 12(9): e0184098. https://doi.org/10.1371/journal.pone.0184098

6. Shao W, Khin S, Kopp WC. Characterization of effect of repeated freeze and thaw cycles on stability of genomic DNA using pulsed field gel electrophoresis. Biopreserv Biobank. 2012;10(1):4-11. doi:10.1089/bio.2011.0016

7. Röder B, Frühwirth $K$, Vogl C, Wagner M, Rossmanith P. Impact of long-term storage on stability of standard DNA for nucleic acid-based methods. J Clin Microbiol. 2010;48(11):4260-4262. doi:10.1128/JCM.01230-10

8. Nakayama Y, Yamaguchi H, Einaga N, Esumi M. Pitfalls of DNA Quantification Using DNA-Binding Fluorescent Dyes and Suggested Solutions. PLoS One. 2016;11(3):e0150528. Published 2016 Mar 3. doi:10.1371/journal.pone.0150528

9. Hsieh AYY, Saberi S, Ajaykumar A, et al. Optimization of a Relative Telomere Length Assay by Monochromatic Multiplex Real-Time Quantitative PCR on the LightCycler 480: Sources of Variability and Quality Control Considerations. J Mol Diagn. 2016;18(3):425-437. doi:10.1016/j.jmoldx.2016.01.004

10. Jiménez KM, Forero DA. Effect of master mixes on the measurement of telomere length by qPCR. Mol Biol Rep. 2018;45(4):633-638. doi:10.1007/s11033-018-4175-y

11. Martin-Ruiz CM, Baird D, Roger L, et al. Reproducibility of telomere length assessment: an international collaborative study. Int J Epidemiol. 2015;44(5):1673-1683. doi:10.1093/ije/dyu191

12. Eisenberg DT, Kuzawa CW, Hayes MG. Improving qPCR telomere length assays: Controlling for well position effects increases statistical power. Am J Hum Biol. 2015;27(4):570-575. doi:10.1002/ajhb.22690

13. Eisenberg DT. Telomere length measurement validity: the coefficient of variation is invalid and cannot be used to compare quantitative polymerase chain reaction and Southern blot telomere length measurement techniques. Int J Epidemiol. 2016;45(4):1295-1298. doi:10.1093/ije/dyw191

14. Verhulst S, Susser E, Factor-Litvak PR, et al. Commentary: The reliability of telomere length measurements [published correction appears in Int J Epidemiol. 2015 Oct;44(5):1749-54]. Int J Epidemiol. 2015;44(5):1683-1686. doi:10.1093/ije/dyv166

15. Verhulst S. Improving comparability between qPCR-based telomere studies. Mol Ecol Resour. 2020;20(1):11-13. doi:10.1111/1755-0998.13114

16. Detry MA, Ma Y. Analyzing Repeated Measurements Using Mixed Models. JAMA. 2016;315(4):407-408. doi:10.1001/jama.2015.19394

17. Benetos $A$, Verhulst $S$, Labat $C$, et al. Telomere length tracking in children and their parents: implications for adult onset diseases. FASEB J. 2019;33(12):14248-14253. doi:10.1096/fj.201901275R 